Title: Architecture Design of Reconfigurable Pipelined Datapaths

Contact Author:

Darren Cronquist

Dept. of Computer Science and Engineering

University of Washington

Box 352350

Seattle, WA 98195-2350

Phone Number: (206) 616-1853

email: darrenc@cs.washington.edu

FAX: (206) 543-2969 


\title{
Architecture Design of Reconfigurable Pipelined Datapaths*
}

\author{
Darren C. Cronquist, Paul Franklin, Chris Fisher, Miguel Figueroa and Carl Ebeling \\ Department of Computer Science and Engineering \\ University of Washington \\ Box 352350 \\ Seattle, WA 98195-2350
}

\begin{abstract}
This paper examines reconfigurable pipelined datapaths (RaPiDs), a new architecture style for computationintensive applications that bridges the cost/performance gap between general purpose and application specific architectures. RaPiDs can provide significantly higher performance than general purpose processors on a wide range of applications from the areas of video and signal processing, scientific computing, and communications. Moreover, RaPiDs provide the flexibility that doesn't come with application-specific architectures.

A RaPiD architecture is optimized for highly repetitive, computationally-intensive tasks. Very deep application-specific computation pipelines can be configured in RaPiDs that deliver very high performance for a wide range of applications. RaPiDs achieve this using a coarse-grained reconfigurable architecture that mixes the appropriate amount of static configuration with dynamic control.

We describe the fundamental features of a RaPiD architecture, including the linear array of functional units, a programmable segmented bus structure, and a programmable control architecture. In addition, we outline the floorplan of the architecture and provide timing data for the most critical paths. We conclude with performance numbers for several applications on an instance of a RaPiD architecture.
\end{abstract}

\section{Introduction}

Many applications from a variety of fields including signal processing, scientific computing, graphics, and communications represent great challenges for today's compiler and architecture designers. Enormous data sets and large computational requirements push compiler and architecture capabilities to the limit. The importance of efficient execution is seen in algorithms such as motion estimation for real-time video encoding and accurate low-power filtering for wireless communications. Such computation-intensive applications have been targeted to a variety of different architectures including general purpose processors, application specific integrated circuits, and field-programmable compute engines. Each of these approaches makes tradeoffs between the range supported applications and performance.

The most flexible architectures are general purpose processors, including the large class of digital signal processors (DSPs). To achieve performance for a wide range of applications, general purpose processors dedicate a substantial amount of die area to data and instruction caches, a crossbar interconnect of the functional units, and complex on-line analyses such as speculative execution and branch prediction. These complex mechanisms can extract a moderate amount of instruction-level parallelism from ordinary programs but are not meant to extract the large amount of fine-grained parallelism available in many compute-intensive applications.

At the other end of the flexibility spectrum lie application specific integrated circuits (ASICs) which have long been used to achieve higher performance at a lower cost than general purpose processors. High performance can be achieved since the architecture can be tailored for a specific application to extract the available fine-grained parallelism, while optimizing for power and/or die area. However, the drawbacks of ASICs are their lack of flexibility and their high non-recoverable engineering costs. By definition, an application specific architecture speeds up only one application. This inflexibility combined with a high design cost make them unattractive except for very well-defined and wide-spread applications. Compounding this problem is the fabrication expense that can reach $\$ 100 \mathrm{k}$, a cost that makes the use of ASICs reasonable only in high volumes.

*This work was supported in part by the DARPA under Contract DAAH04-94-G0272, and in part by Tektronix. D. Cronquist was supported in part by a Gray fellowship. P. Franklin was supported in part by an NSF fellowship and in part by an Intel Foundation fellowship. 
Field programmable computing has attracted a lot of attention recently because of its promise to bridge the flexibility and performance gaps between general purpose processors and ASICs. A field-programmable architecture is like an electronic lego construction set. The user puts legos (called logic blocks) together to form a circuit that best suits his current application. At any time, the user can construct a new circuit by taking apart the old one and building from scratch. As a result, configurable computing machines can deliver, in theory, the high performance provided by application specific hardware along with the flexibility of general purpose processors. Unfortunately, this promise has yet to be realized in spite of some successful examples [1, 11]. There are two main reasons for this.

First, configurable computing platforms are currently implemented using commercial field programmable gate arrays (FPGAs). FPGAs are necessarily very fine-grained (i.e. all of the logic blocks are small and regular) so they can be used to implement arbitrary circuits, but the overhead of this generality is expensive in both area and performance. While general purpose processors use highly optimized functional units that operate in bit-parallel fashion on long data words, FPGAs are very inefficient for ordinary arithmetic and only somewhat better for logical operations. FPGA-based computing has an area/performance advantage only on complex bit-oriented computations or complicated bit-level masking and filtering.

Second, programming an FPGA-based configurable computer is akin to designing an ASIC. The programmer either uses synthesis tools that deliver poor density and performance or designs the circuit manually, which requires both intimate knowledge of the FPGA architecture and substantial design time. Neither alternative is attractive, particularly for simple computations that can be described in a few lines of $\mathrm{C}$ code.

Our response to these problems is a new architecture style - reconfigurable pipelined datapaths (RaPiDs). RaPiDs are coarse-grained field-programmable architectures for constructing deep computational pipelines. As compared to a general purpose processor, a RaPiD can be thought of as a superscalar architecture with hundreds of functional units but with no cache, register file, or crossbar interconnect. Instead of a data cache, data is streamed in directly from external memory or sensors. Instead of an instruction cache, programmed controllers generate a small instruction stream which is decoded as it flows in parallel with the datapath. Instead of a global register file, data and intermediate results are stored locally in registers and small RAMs, close to their destination functional units. Instead of a crossbar, a programmable interconnect is configured to forward data between specific functional units on a per application basis.

Removing caches, crossbars, and register files frees up a tremendous amount of area that can be dedicated to compute resources, and reduces the communication delay by shortening wires. Unfortunately, these removals also reduce the types of applications that can be computed on RaPiDs. Highly irregular computations, with complex addressing patterns, little reuse of data, and an absence of fine-grained parallelism will not map well to a RaPiD architecture. However, regular computation-intensive tasks like those found in digital signal processing, scientific computing, graphics, and communications will reap great performance gains on RaPiDs over general purpose processors. In addition, a RaPiD provides the flexibility that doesn't come with an ASIC.

The fundamental architectural features of RaPiDs have been developed in parallel with a programming language and compiler since a strong synergy exists between architecture, specification, and compilation. The user must be able to easily take advantage of the full capabilities of the architecture. In addition, the architecture must have facilities for easing the compilation of a given specification. To this end, specialized hardware is added to the architecture and special constructs are added to the language with the ultimate goal of making specification simple, compilation feasible, and hardware utilization high. Details of specification and compilation can be found in [3].

This paper describes the RaPiD architecture in detail. The block diagram in Figure 1 breaks RaPiDs down into a datapath, a control path, an instruction generator, a stream generator, and a memory interface. This paper discusses the fundamental features of each of these components. In particular, Section 2 introduces the datapath architecture, including how functional units and buses are used to form an application's datapath. Section 3 presents a novel architecture for the generation of control for the datapath, including details on the programmed controller architecture for instruction generation. Section 4 discusses how RaPiD interfaces with external memory through the stream generator. Finally, Section 5 introduces a benchmark architecture, and Section 6 analyses area, clock rate, and power requirements of this architecture in a specific technology.

\section{The Datapath Architecture}

A typical RaPiD datapath contains hundreds of functional units, ranging in complexity from a simple general purpose register to a multi-output booth-encoded multiplier with a configurable shifter. The ability of the architecture to forward results between these functional units determines, in part, the range of applications that can be targeted. The interconnection method employed by many of today's superscalar processors is a crossbar- 


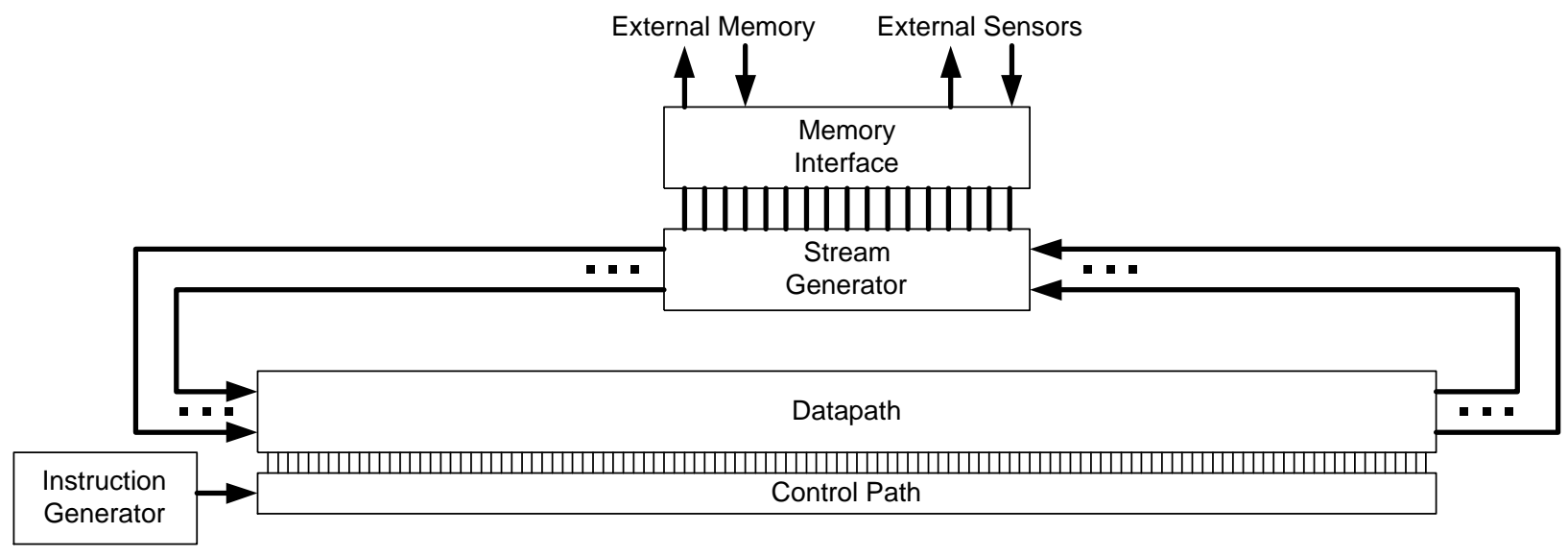

Figure 1: RaPiD Architecture Block Diagram. The RaPiD datapath is a linear pipeline that is configured from a linear array of functional units by means of a programmable interconnect structure. The instruction generator produces a stream which is decoded by the control path. The resulting decoded instructions provide time-varying control for the datapath. The stream generator communicates with external memory (or memory-mapped external sensors) to stream data in and out of the RaPiD datapath.

a completely flexible interconnect (any functional unit can forward results to any other) which greatly simplifies instruction scheduling ([7]). Unfortunately, the chip area of the crossbar scales with the square of the number of functional units. To avoid this area expense, RaPiD borrows from FPGA architectures by interconnecting its functional units via a programmable, segmented bus structure.

Since RaPiDs consist of coarse-grained, word-based functional units, the interconnect differs from that of FPGAs. The buses are word-based instead of bit based and are arranged linearly as opposed to two-dimensionally. A linear structure is easily manageable; it simplifies the layout and reduces the control requirements. Functional units can be more tightly spaced, and there is no need for corner turning switches. Moreover, a wealth of research exists showing how multidimensional algorithms can be mapped to linear arrays $[6,10,8]$, especially if some memories are embedded in the datapath since local memory can act like an extra dimension for deeply nested specifications. The linear structure of the RaPiD datapath is shown in Figure 2.

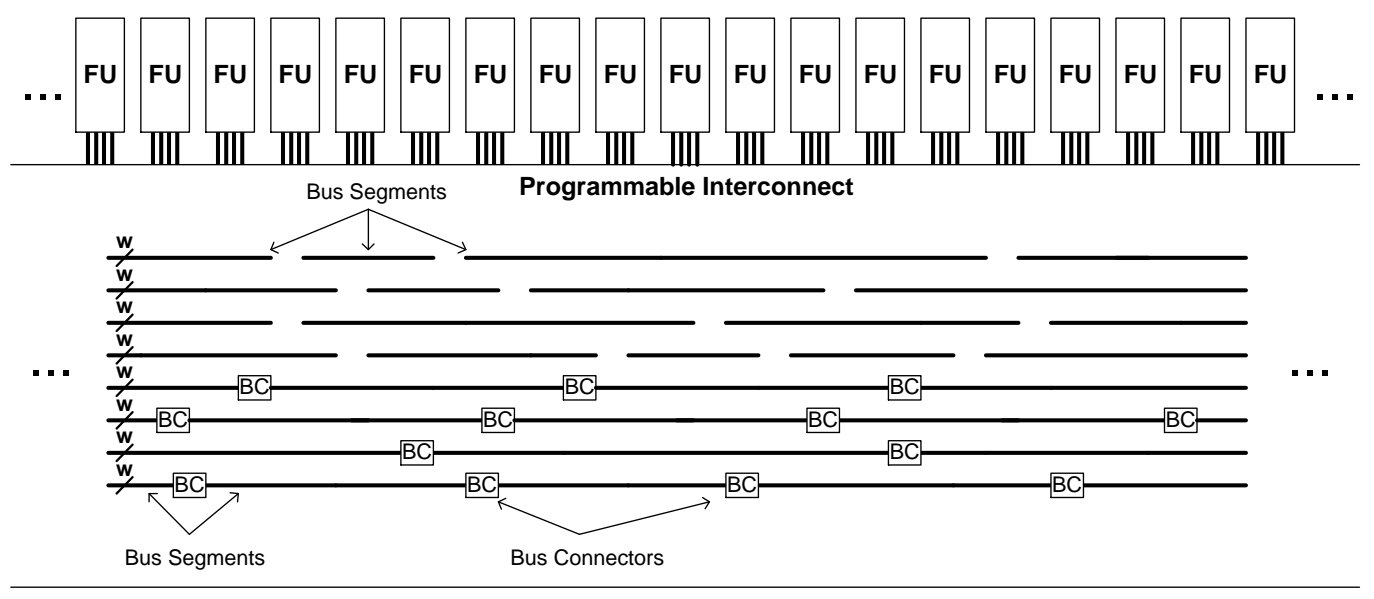

Figure 2: Overview of the RaPiD Datapath. Functional units are arranged linearly over a programmable, segmented bus structure. A set of $B-1$ tracks run the entire length of the datapath. Some tracks contain bus connectors to make configurable length buses.

RaPiDs are targeted at word-based computations. The data-width, as well as the choice of fixed-point or floating-point, are architectural design parameters. In most cases, the fixed-point data width is between 8 and 32 bits, and both signed and unsigned data are supported. Multiple fixed-point representations (within the same 
width) can be provided by configurable shifters in the datapath. For example, it is often necessary to have a shifter follow a multiplier to allow the correct fixed-point representation to be chosen for the multiplier output.

Operation errors like overflow cannot be handled as in normal processors. Instead, an extra tag bit is associated with each data value. The functional units can be configured to set the tag when an overflow occurs. The tag could be used to represent an error state which would be propagated through all future computations. Alternatively, the tag could indicate a saturated value, allowing for further computation.

\subsection{Functional Units}

Each functional unit inputs a set of words from the datapath, performs a computation based on set of control bits, and outputs results in the form of data words and status bits. The status outputs allow for data-dependent control to be generated. A generic functional unit is shown in Figure 3a. All functional unit outputs pass through a ConfigDelay unit which can be configured as 0 to 3 register delays, as shown in Figure 3b. These optional registers allow for the creation of very deep pipelines.

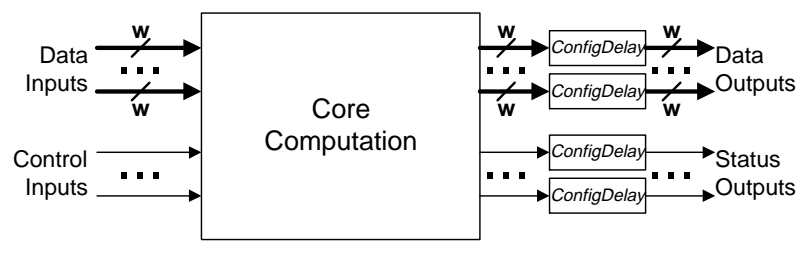

(a)

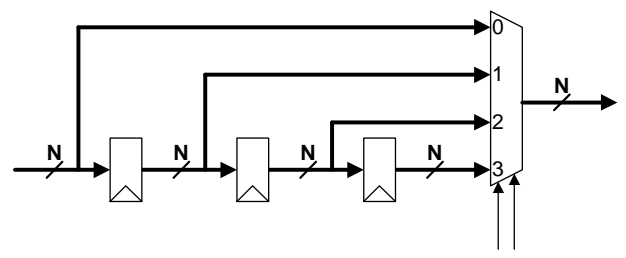

(b)

Figure 3: A Generic Functional Unit and a Configurable Delay. (a) Each functional unit has inputs and outputs for both data and control. Each output passes through a ConfigDelay unit. (b) A ConfigDelay unit's N-bit output is equivalent to its input delayed by up to 3 registers, as determined by the two multiplexer control bits.

A variety of functional units can be included in a RaPiD architecture. General-purpose functional units like ALUs, multipliers, shifters and memories are the most common, but for specific domains, a special-purpose functional unit which performs a single function (i.e. has no control inputs) might make the most efficient use of silicon. An example is a Viterbi decoder for communication applications. For other domains, a highly configurable functional unit might be the right choice. For example, a functional unit could be constructed of FPGA-like logic blocks to support a range of bit manipulations like find first one, count ones, and normalize.

Memories within the datapath provide space for temporary variables, constant tables and configurable-length delay lines. The size of memory is an implementation parameter. Our experience has shown that for applications we have programmed, a local memory with $3 N$ entries is sufficient for pipelines with $N$ cells. Section 5 examines several functional units that are used in the benchmark architecture.

\subsection{Programmable Interconnect}

The programmable interconnect consists of a set of $B-1$ segmented tracks ${ }^{1}$ that run the entire length of the datapath, as shown in Figure 2. Each track contains a set of bus segments, some of which are connected by bus connectors. All buses have the same width, which matches the data width operated on by the functional units. Some functional units may require or produce double-width data values, which are communicated via two buses. These values can be treated as two independent single-width values and routed independently, for example, to two different ALUs for double-precision accumulation.

An input to a functional unit can be zero (GND) or any one of the $B-1$ tracks from the interconnect. To accomplish this, each data input is driven by a $B: 1$ multiplexer, whose $\lceil\lg B\rceil$ select lines are driven by control signals as shown in Figure 4. The zero input can be used, for example, to clear registers.

$\mathrm{RaPiD}$ allows each functional unit output to drive an arbitrary number of buses via $B-1$ tristate drivers which are configured using $B-1$ control bits. Since each tristate driver is configured independently, an output can fan out to several buses or none at all if the functional unit is unused.

A RaPiD datapath is usually divided into identical units, called cells, which are replicated to form a complete datapath. For example, the simple cell shown in Figure 5 consists of three 1-input functional units without control (FU1s), three 2-input functional units with control (FU2s), seven tracks, and six bus connectors. The

\footnotetext{
${ }^{1}$ We used $B-1$ here to be consistent with the B-input multiplexers (GND is an additional input).
} 


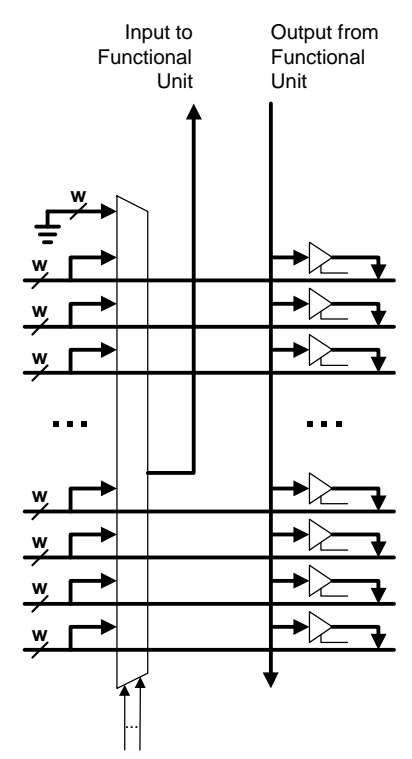

(a)

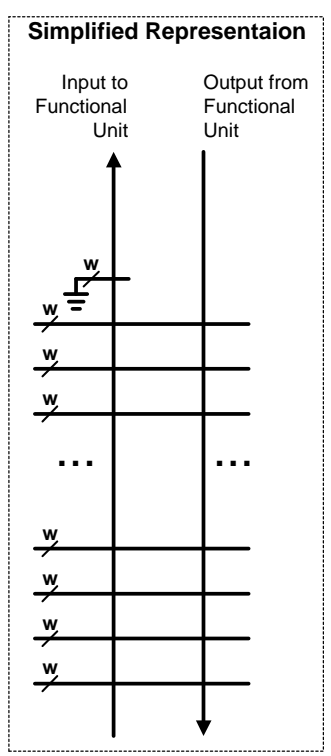

(b)

Figure 4: Interconnect Between Functional Units and Buses. (a) Each data input uses a $B: 1$ multiplexer to select a bus. Each data output uses $B-1$ tristate drivers to select the buses to drive. (b) This equivalent but simplified representation uses a single line to represent a multiplexer for inputs and tristate drivers for outputs.

number of replications performed to complete the datapath depends on the target technology and the application domain's performance requirements. This division of the datapath into cells is invisible when it comes to mapping applications.

The first several tracks of the interconnect have bus segments of differing lengths to allow for many types of data forwarding. However, having fixed-length bus segments in all tracks is too restrictive for many situations. For example, a pipelined bus carrying data across the entire array is very common. Moreover, the mapping problem simplifies when the lengths of the bus segments can be varied from application to application. To support configurable length segments, several of the tracks are populated with bus connectors (Figure 5b). A bus connector can drive left, drive right, or be disconnected. The connection is buffered to reduce delay in high fanout signals and can also be configured to provide up to three register delays.

\section{The RaPiD Control Architecture}

The previous section focused on the architectural requirements for interconnecting a RaPiD's functional units. A specific interconnect is determined by the many control bits found in the multiplexers, tristate drivers, ConfigDelay units, bus connectors, and functional units. Table 1a examines the control requirements for the cell of Figure 5a. Since there are seven tracks, each multiplexer requires three control bits, and each functional unit data output requires seven tristate drivers. Each bus connector and every functional unit output (data and control) have a ConfigDelay unit which requires two control bits. The total number of control bits required for this example is 117. Table $1 \mathrm{~b}$ shows that a single RaPiD-Benchmark cell requires 393 control bits, and hence a 16-cell datapath for would require 6288 bits.

There are several approaches for generating the control for this architecture. The most straightforward method is to treat all control as field-programmable configuration bits like in an FPGA. Unfortunately, this approach is too inflexible and only applies to algorithms that can be mapped to a purely static dataflow network. Any application that requires, for example, a register to be cleared, a RAM address to be incremented, or an ALU operation to be changed on a specific cycle needs control that can change on each cycle. Another approach for control generation is a programmed control architecture with a $6 \mathrm{~Kb}$ wide instruction stream. Unfortunately, generating such a wide instruction on every cycle would be prohibitively expensive.

Our solution is to divide the control into hard control, which is fixed for the duration of an application, and 


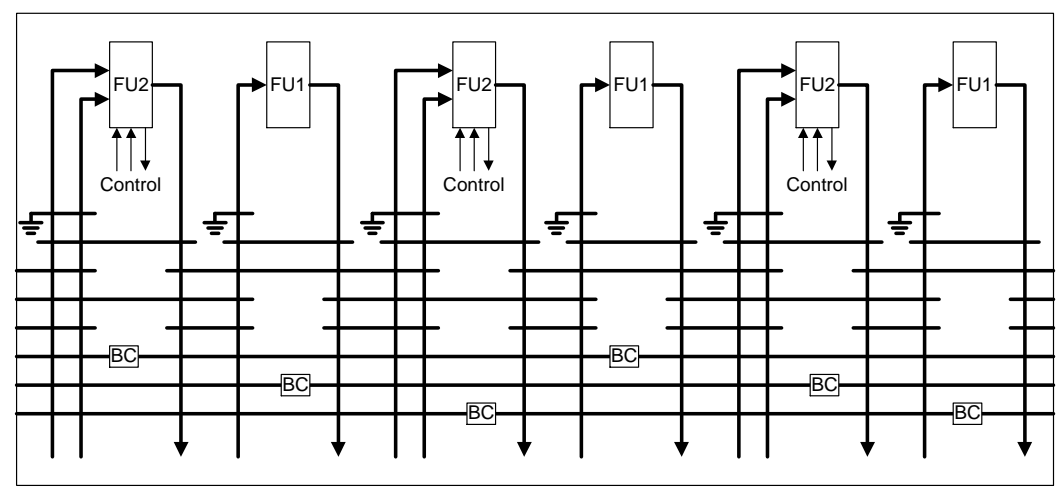

(a)

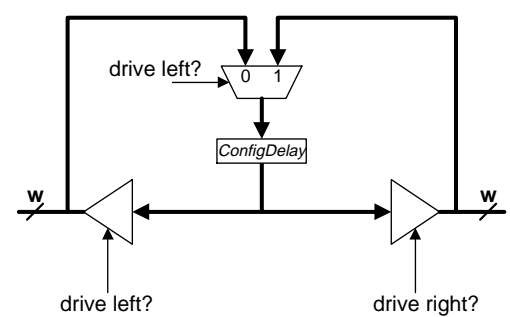

(b)

Figure 5: RaPiD Datapath Cell. (a) This example cell has seven tracks, six functional units, and six bus connectors. Replicas of this cell are cascaded horizontally to form the entire datapath. (b) A bus connector can drive left, right, or be left unconnected. It can be used to buffer the signal between adjacent bus segments or to create up to three register delays via the ConfigDelay unit.

Table 1: Control Requirements. (a) The example cell in Figure 5 requires 117 control bits. (b) The RaPiD-Benchmark cell from Section 5 requires 393 control bits.

(a)

\begin{tabular}{||l|r|r|r||rr||}
\hline Unit & $\frac{\text { Bits }}{\text { Unit }}$ & $\frac{\text { Units }}{\text { cell }}$ & $\frac{\text { Bits }}{\text { cell }}$ & Soft & Hard \\
\hline Multiplexer & 3 & 9 & 27 & 27 & 0 \\
Tristate Driver & 1 & 42 & 42 & 0 & 42 \\
ConfigDelay & 2 & 15 & 30 & 0 & 30 \\
Bus Connector & 2 & 6 & 12 & 0 & 12 \\
FU1 & 0 & 3 & 0 & 0 & 0 \\
FU2 & 2 & 3 & 6 & 6 & 0 \\
\hline Total & & & 117 & 33 & 84 \\
\hline
\end{tabular}

(b)

\begin{tabular}{||l|r|r|r||rr||}
\hline Unit & $\frac{\text { Bits }}{\text { Unit }}$ & $\frac{\text { Units }}{\text { cell }}$ & $\frac{\text { Bits }}{\text { cell }}$ & Soft & Hard \\
Multiplexer & 4 & 20 & 80 & 80 & 0 \\
Tristate Driver & 1 & 196 & 196 & 0 & 196 \\
ConfigDelay & 2 & 27 & 54 & 0 & 54 \\
Bus Connector & 2 & 14 & 28 & 0 & 28 \\
GP Register & 0 & 6 & 0 & 0 & 0 \\
ALU & 6 & 3 & 18 & 18 & 0 \\
RAM & 3 & 3 & 9 & 6 & 3 \\
Multiplier & 8 & 1 & 8 & 0 & 8 \\
\hline Total & & & 393 & 104 & 289 \\
\hline
\end{tabular}

soft control, which can change on every cycle. A small percentage of the control bits in a RaPiD architecture need the flexibility of soft control. By making the multiplexer control bits soft, the tristate driver control can be made hard. This retains the ability to perform dynamic data forwarding and avoids the extra delay introduced by dynamically controlled tristate drivers. In addition, the ConfigDelay unit's control is hard since the amount of pipelining in the datapath tends to be fixed over the course of an application. Functional units divide their control between hard and soft depending on the required flexibility. The final two columns of Table 1 divide the control into soft and hard bits for both the example cell and the RaPiD-Benchmark cell.

As seen in Table 1, approximately $25 \%$ of the a RaPiD's control is soft and the remaining $75 \%$ are fieldprogrammable via SRAM bits as in an FPGA. However, only if the soft control can be generated efficiently, in terms of area and speed, will applications be able to reap the performance benefits of a RaPiD architecture.

A RaPiD's application domain consists of pipelined computations which are very repetitive. Apart from initialization, finalization, and boundary processing, algorithms spend most of their time in deeply nested computation kernels. Such applications are naturally represented by the composition of deeply nested loops (see [3]). Soft control is statically compiled from the nested loop specification. The challenging requirements of soft control are low instruction bandwidth and fast instruction generation.

Figure 6 examines possible control architectures. The first method is to convert the required soft control into a set of state machines, which could then be mapped to an FPGA. This approach, shown in Figure 6a, can take advantage of traditional synthesis techniques for optimizing control. However, this is a more inefficient approach than using dedicated counters and comparators, as found in the programmed controller approach of Figure $6 \mathrm{~b}$ since much of the control state comes from a nested loop specification. Unfortunately, a controller with a very long instruction is also expensive in terms of area and would most likely be the performance bottleneck due to 
overheads in synchronization and instruction bandwidth.
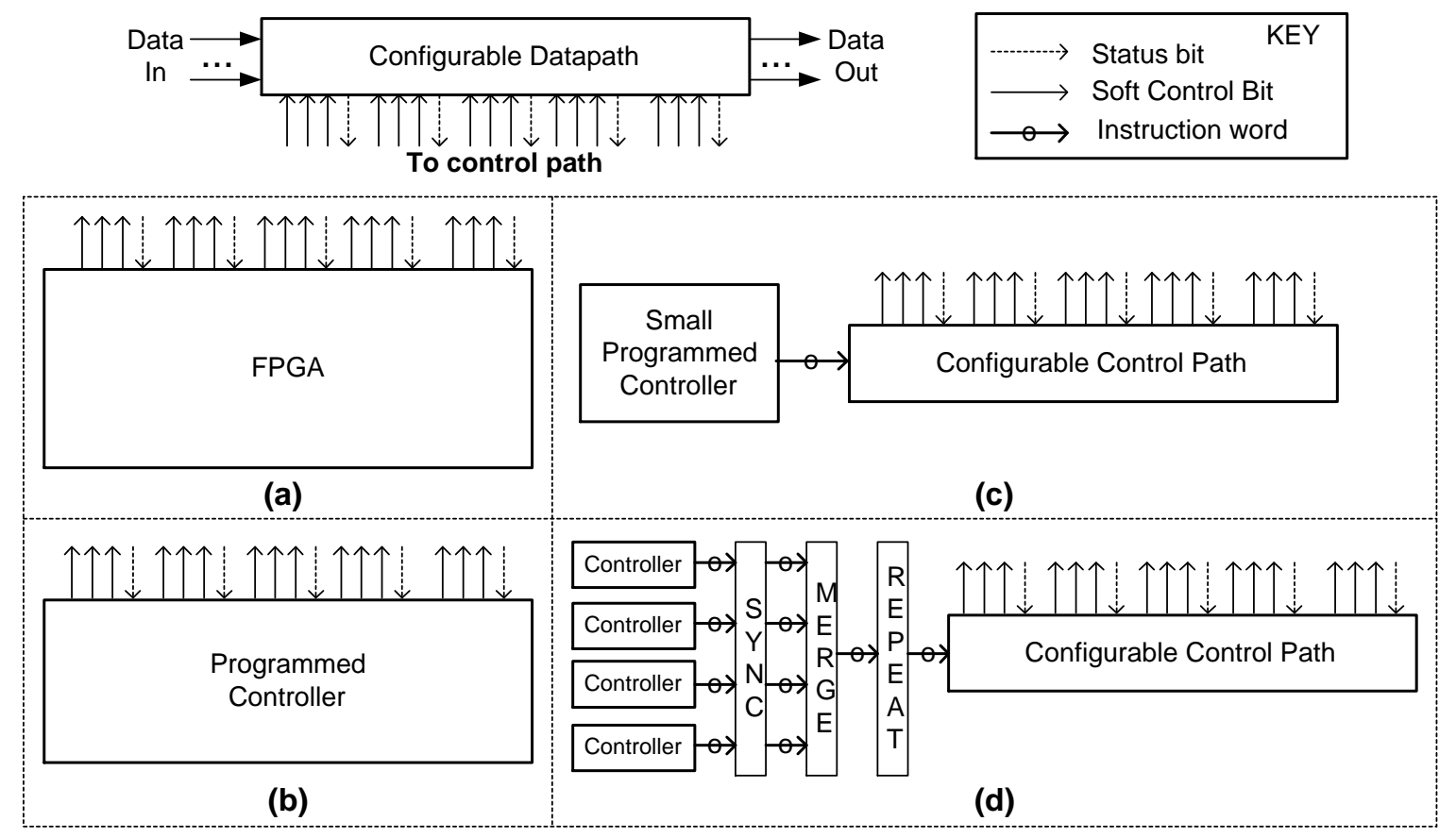

Figure 6: Soft Control Implementation Options. (a) State machines are mapped to an FPGA. (b) A programmed controller generates a VLIW. (c) A programmed controller generates a short instruction which is decoded by a configurable path. (d) The controller is broken down into multiple, parallel programmed controllers which provide better support for parallel loop specifications.

The length of the instruction can be greatly reduced by making two key observations. First, most of the soft control is actually constant for a particular application. Second, because of the regularity of computations, much of the dynamic soft control can be used to control more than one operation in more than one pipeline stage. As a result, our approach for soft control generation is to use a smaller programmed controller with a short instruction along with a configurable path containing a limited number of buses and logic gates, as shown in Figure 6c. The controller generates instruction bits by executing code derived from the application's loop structure. The configurable path uses these instruction bits, along with status bits from the functional units, to form the soft control.

The configurable control path of Figure 6c looks like a scaled down version of the RaPiD datapath in Figure 2. A set of configurable logic blocks are interconnected via a segmented bus structure which runs parallel to the datapath. Each track can be driven by any bit of the instruction word. These bits then flow in parallel to the datapath and potentially through logic blocks in order to produce the required soft control signals. The complexity of the logic blocks depends on the architecture's application domain and is typically some sort of look-up table.

In addition to logic blocks, each soft control signal can be optionally inverted. The optional inverter requires a hard control bit to select the true or inverted signal and has optional registers on its output. If a soft control signal is constant for the duration of an application, GND is selected as the input, and the optional inverter is configured to output a 0 or 1 .

As in the datapath, a set of segmented tracks run the extent of the array. The number of tracks required in the control path varies by application but is not large because control signals tend to be reused extensively. Interconnecting configurable logic blocks and optional inverters is done with multiplexers and tristate drivers, as was shown for the datapath in Figure 4.

Figure 7 extends the example of Figure 5a for control. A cell of control is shown that generates the 33 soft control bits. Each cell has one logic block, implemented as a 3-input look-up-table (3-LUT). A total of seven tracks are used. The optional inverter structure is shown in Figure $7 \mathrm{~b}$. 


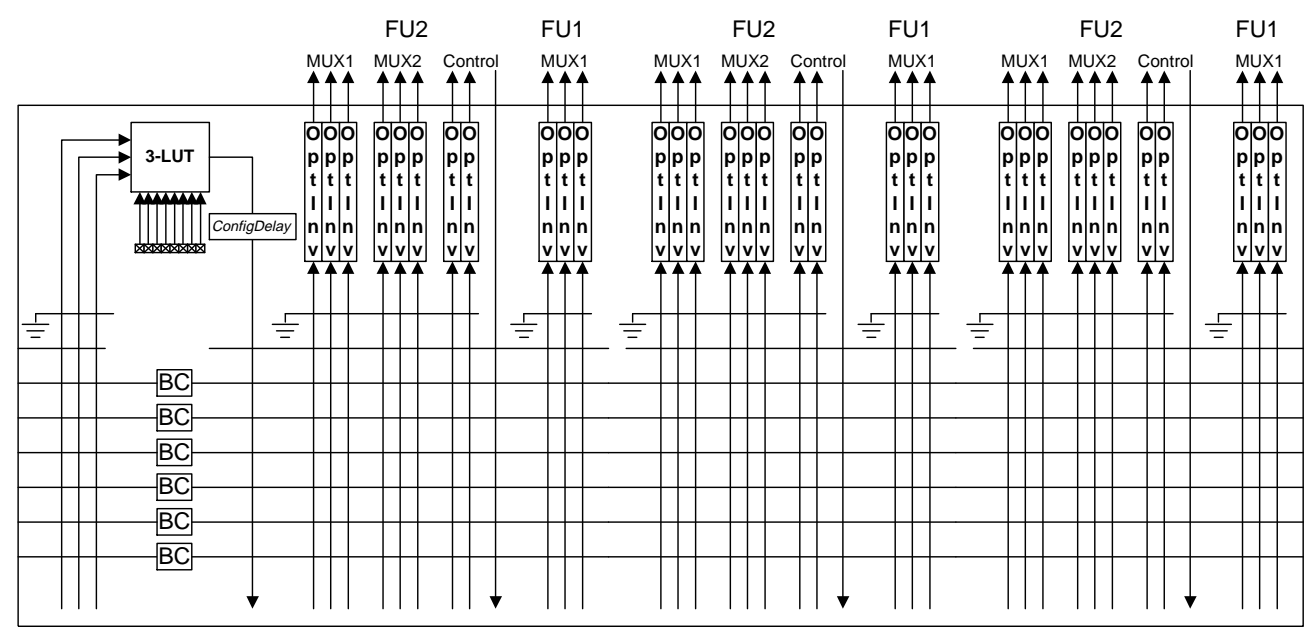

(a)

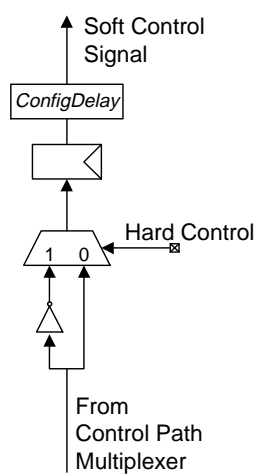

(b)

Figure 7: RaPiD Control Path Cell. (a) This example cell produces the control signals required for Figure 5. There are seven tracks, one logic block, and six bus connectors. Replicas of this cell are cascaded horizontally to form the entire control path. (b) An optional inverter drives each soft control signal. It takes a signal from the control path, optionally inverts it, and registers the result. The ConfigDelay unit allows for up to 3 additional register delays.

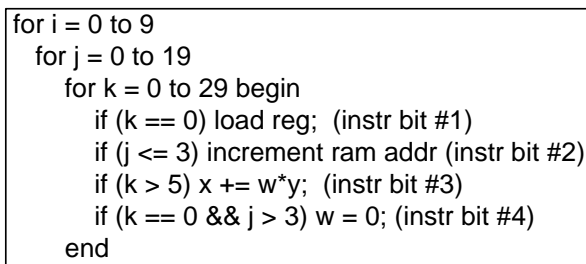

(a)

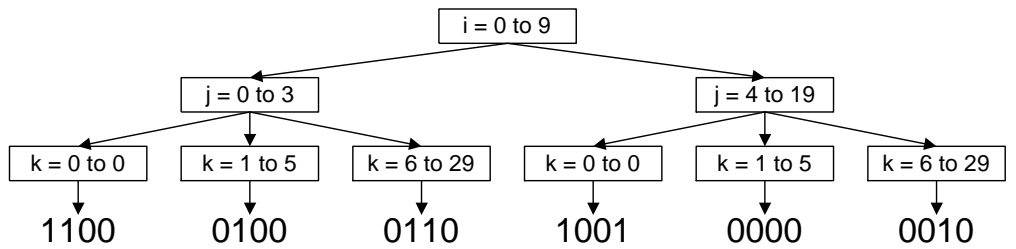

(b)

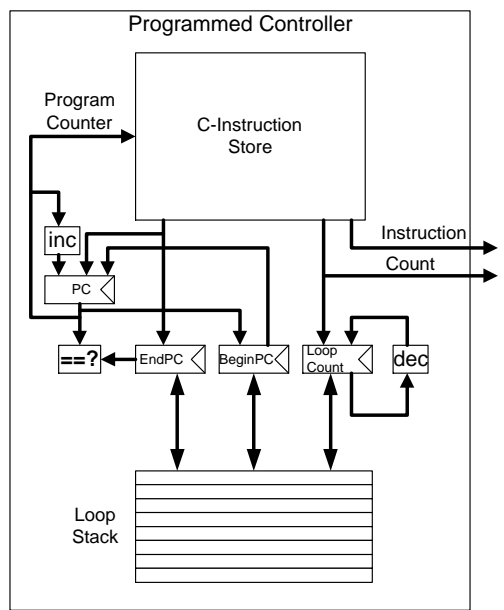

(d)

\begin{tabular}{|c|c|c|c|c|}
\hline 1 & & loop & 10 & end1 \\
\hline 2 & & loop & 4 & end 2 \\
\hline 3 & & inst & 1 & 1100 \\
\hline 4 & & inst & 5 & 0100 \\
\hline 5 & end 2 : & inst & 24 & 0110 \\
\hline 6 & & loop & 16 & end 1 \\
\hline 7 & & inst & 1 & 1001 \\
\hline 8 & & inst & 5 & 0000 \\
\hline 9 & end1: & inst & 24 & 0010 \\
\hline 10 & & halt & & \\
\hline
\end{tabular}

(c)

Figure 8: Programming a Controller. (a) Each line in the code corresponds to an instruction bit. (b) The loop nest is statically compiled producing a loop nest with instruction words. (c) The loop nest is converted to C-instructions. (d) This programmed controller is optimized for the execution of nested loops. 


\subsection{Instruction Generator}

The instruction word of Figure 6c consists of bits derived from the application's nested loop specification. These instruction bits are generated by a programmed controller which is optimized to execute nested loop structures. The algorithm is first statically compiled to yield a program for this controller. For example, consider the nested loop code of Figure 8a. Each of the four conditional statements generates one bit of the instruction. Static compilation removes the conditionals on the loop variables, expanding this loop to generate static instructions as shown in Figure 8b.

The programmed controller in Figure 6c must be able to execute loop structures like the one in Figure 8b and produce, on average, at least one instruction per cycle. To avoid the complexity of avoiding stall cycles when dealing with the boundary cases of loop nests, the controller packs innermost loops into a single instruction with a count and provides a repeater to issue instructions the appropriate number of times.

Instructions executed by the programmed controller are called $C$-instructions. The C-instruction "inst CNT I" is used to output the instruction word "I" to the control path "CNT" consecutive times. The C-instruction "loop CNT LAST" executes a loop "CNT" times, starting at the next program counter and ending at "LAST". For example, Figure 8c shows a set of C-instructions equivalent to the code in Figure $8 \mathrm{~b}$.

The programmed controller design is shown in Figure 8d. A loop stack is used to optimize handling of nested loops. Each time the controller encounters a "loop" C-instruction, it fills the LoopCount, BeginPC, and EndPC registers and pushes any prior loop data onto the loop stack. The controller then executes the loop body until EndPC $==$ PC. Then, PC is replaced with BeginPC and LoopCount is decremented. When LoopCount equals one, the loop stack is popped, forcing the controller to fall through after the last iteration of the loop body. This specialized implementation requires only one cycle for loop initialization; the remainder of the loop processing is isolated in the loop stack and program counter and is overlapped with executing the loop body. This compares favorably to more typical loop approaches where looping instructions appear in the loop body itself.

Even though a single programmed controller is sufficient, it is not the best match for a specification consisting of parallel loop nests. For example, to implement the loop nest of Figure 8a running in parallel with another loop nest, a single controller would have to take, in the worst case, the cross-product of the two loops nests to generate all instructions words. A more efficient approach is to have multiple controllers running in parallel, one per parallel loop nest, as shown in Figure 6d. Synchronization between parallel loops is done via signal/wait synchronization primitives. A synchronization unit watches the flow of C-instructions from all controllers and aligns the streams according to the signal/wait pairs. The C-instruction "signal NUM" is a non-blocking operation tells controller number "NUM" to stop waiting or to skip its next wait if not currently waiting. The C-instruction "wait I" simply repeats instruction word "I" until a signal arrives.

Unfortunately, instruction bits that depend on variables across controllers may require the use of additional logic gates in the configurable path. To reduce this gate requirement, the outputs of all controllers are merged to form a single instruction stream, as shown in Figure 6d. The merge unit could be a configurable PLA-type structure or a simple bitwise-OR if less complexity is needed.

\section{RaPiD Memory Architecture}

Within a RaPiD architecture, memory accesses are decoupled from the instruction stream. The sequences of memory references from the nested loop specification are mapped to address generators, as shown in Figure 9. Each address generator is associated with a FIFO, forming an input or an output stream. Using the addresses from the address generators, input stream FIFOs are filled from memory and output stream FIFOs are emptied to memory. These reads and writes are handled by a memory interface which routes addresses and data between the streams and external memory modules. The memory must provide high bandwidth either through fast SRAM memory technology, aggressive interleaving, and/or batching and out-of-order handling of addresses. Applications typically execute between 1 and 3 operations per cycle so that the memory must sustain a data transfer of up 3 words/cycle. The memory interface also provides a memory-mapped mechanism to stream data from external sensors instead of external memory.

The address generators closely resemble the programmed controller in Figure $8 \mathrm{~d}$ but produce addresses instead of instructions. In order to output more than one address per cycle on average, addresses are packaged with a count and a stride. The repeater in Figure 9 differs from the repeater in Figure $6 \mathrm{~d}$ since it must increment the address by the stride on each repeat.

The addressing pattern for each stream is statically determined at compile time. Reads and writes to the stream FIFOs are controlled from instruction bits in the control path, as illustrated in Figure 9. Synchronization between the data and instruction streams is achieved by halting the RaPiD array when a data FIFO is empty on a read or full on a write. 


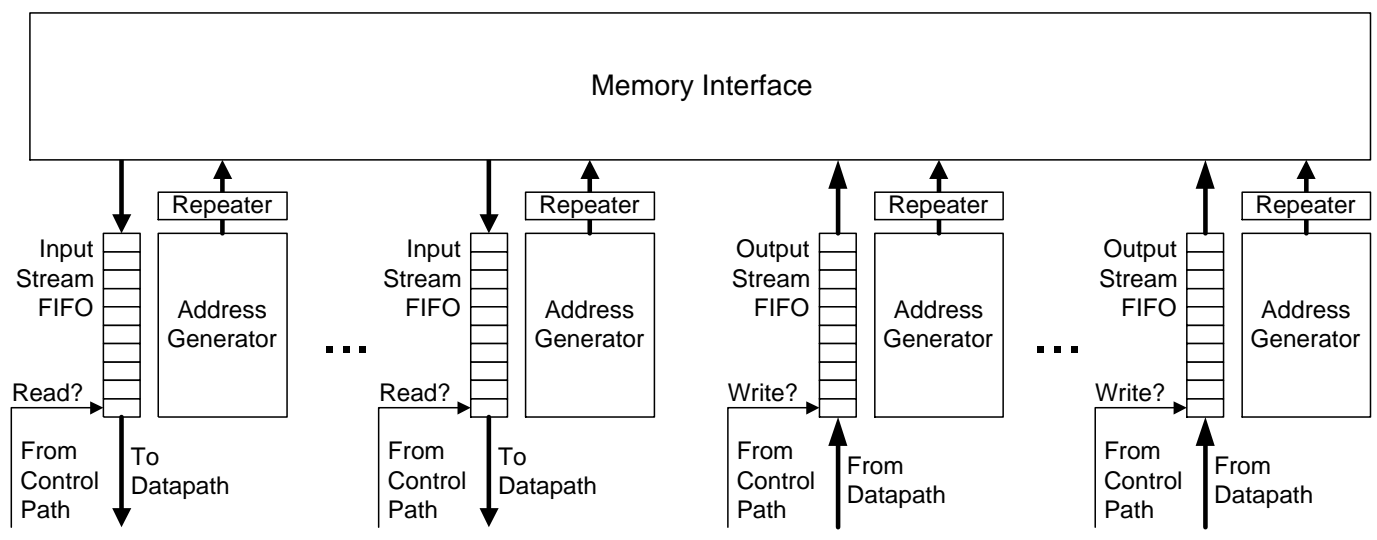

Figure 9: A RaPiD Stream Generator. Each stream FIFO is associated with an address generator. The address generator produces a stream of addresses which are serviced by the memory interface. The corresponding data is then put into or taken from the appropriate FIFO.

\section{$5 \quad$ A Benchmark Architecture}

The previous sections discussed architectural features that apply to all RaPiDs. This section develops a specific RaPiD architecture, called RaPiD-Benchmark, as an illustrative example. We will present cost, performance and power results using this benchmark architecture.

RaPiD-Benchmark's application domain consists primarily of signal processing applications. Such a domain often requires very high precision multiply-accumulates operations, and hence RaPiD-Benchmark has a 16-bit fixed-point datapath with multipliers and ALUs. In addition, the datapath provides general purpose registers and RAMs. A RaPiD-Benchmark cell comprises three ALUs, three 64-word RAMs, six general purpose registers, and one multiplier. There are 14 data tracks and 32 control tracks. This cell is replicated 16 times to form the complete RaPiD-Benchmark datapath. This mix of functional units and pipeline depth was chosen based on the requirements of a range of signal processing applications.

RaPiDs contain an abundance of registers, most of which are used to pipeline the computation. Often a more flexible register is required to store constants and/or temporary values and to forward values from a bus segment in one track to a segment in another track. A functional unit consisting solely of a configurable delay provides this flexibility. We call such a functional unit a general purpose register (GP register).

The most commonly used functional unit is the general-purpose arithmetic logic unit (ALU). Multiple ALUs can be combined in a pipelined way to compute a multiple-width operation, most typically as a 32-bit add for multiply-accumulate computations. The output register of the ALU can also be used as the accumulator for multiply-accumulate operations.

RaPiD-Benchmark includes three local memories per cell. The RAM address is supplied either by the datapath or by a local address generator that supports simple sequential memory access. If values are read and written sequentially, as is the most common case, then no datapath resources need to be used for address generation. Some applications use the RAMs as a configurable-length shift register that delays the values by a fixed number of clock cycles. This is implemented by allowing a read followed by a write to the same address in one clock cycle.

The multiplier unit is a two stage booth encoded multiplier which takes two 16-bit inputs and produces a 32bit result. The result can be shifted by a statically programmed amount to maintain the appropriate fixed-point representation. Both halves of the result are available as output via separate sets of bus drivers.

The instruction generator is comprised of four programmed controllers, a synchronizer, and a simple OR merge unit. There are three input and three output streams, each containing an address generator.

Configuration memory is implemented as a static RAM organized into words of 16 bits each. Words in this RAM can be written in any order so that reconfiguration can be streamlined. To avoid driving buses with two different bus drivers during reconfiguration, each bus has an associated daisy-chained priority signal that ensures at most one driver is enabled at any time. This low-overhead mechanism simplifies and speeds up the reconfiguration process. 


\section{Performance}

This section analyzes the performance, area cost and power consumption of the RaPiD-Benchmark architecture presented in the previous section. This analysis is based on the layout of the components of the RaPiD-Benchmark cell. This layout uses a vintage-1995 3.3v 0.5 $\mu$ CMOS process using MOSIS scalable submicron design rules. These components were fabricated through MOSIS using two test chips. Tests of those chips substantiated the performance results presented here.

We first present the delays for the components of the RaPiD-Benchmark cell and show that a $100 \mathrm{MHz}$ clock is feasible for a scalable pipeline in this technology. The performance results we present later for various applications are based on this $100 \mathrm{MHz}$ clock. We next present the area of these components and the entire RaPiD-Benchmark cell to show the relative sizes of the computing structures and the configuration hardware. Finally, we present estimates of the power consumption of the RaPiD-Benchmark cell.

\subsection{RaPiD Performance}

In order to achieve a $100 \mathrm{MHz}$ clock rate, the longest combinational delay in the datapath must not exceed 10ns, including the register setup time. The delay of various components is given in Table $2 \mathrm{a}$ as measured by HSpice on the layout. (Multiply1 and Multiply2 refer to the first and second pipeline stages of the multiplier.) The In $\rightarrow$ Clk

Table 2: Timing Data. (a) Component Delays (ns). (b) Critical Path Delays (ns).

(a)

\begin{tabular}{||l|c|c|c||}
\hline & \multicolumn{2}{|c||}{ Pipelined } & Comb. \\
\hline Unit & In $\rightarrow$ Clk & Clk $\rightarrow$ Out & Bypass \\
\hline Multiply1 & 6.3 & 1.2 & - \\
Multiply2 & 5.3 & 1.6 & 6.9 \\
ALU & 4.3 & 1.6 & 5.9 \\
Ram & 2.0 & 3.4 & - \\
Bus Connector & 0.5 & 1.2 & 1.6 \\
Data Register & 1.1 & 1.6 & 2.1 \\
Optional Inv. & 1.6 & 1.2 & - \\
3-LUT & 2.0 & 1.2 & 2.2 \\
\hline
\end{tabular}

(b)

\begin{tabular}{||l|c||}
\hline Path & Delay \\
\hline Register $\rightarrow$ four busses $\rightarrow$ Register & 7.5 \\
Register $\rightarrow$ bus $\rightarrow$ Multiply1 & 7.9 \\
Multiply $\rightarrow$ Multiply2 & 6.5 \\
Register $\rightarrow$ two busses $\rightarrow$ ALU & 7.5 \\
Ram $\rightarrow$ one bus $\rightarrow$ ALU & 7.7 \\
Multiply2 $\rightarrow$ two busses $\rightarrow$ Ram & 6.8 \\
Control Register $\rightarrow$ one bus $\rightarrow$ & \\
3LUT $\rightarrow$ two busses $\rightarrow$ Optional Inv. & 6.6 \\
\hline
\end{tabular}

delay is the combinational delay of the component from the input bus segment to the register, including the input multiplexer and the setup time of the register. The $\mathrm{Clk} \rightarrow$ Out delay is the combinational delay of the component from the register to the output bus segment, including the register propagation delay and delay driving the bus. The combinational bypass column gives the delay from the input bus segment to the output bus segment when no register is used.

Table $2 \mathrm{~b}$ gives a number of register-to-register paths whose delays are less than $8 \mathrm{~ns}$. This shows that the RaPiDBenchmark architecture will run at $100 \mathrm{MHz}$, with a timing margin of $25 \%$, as long as applications can be pipelined, placed, and routed within this path delay constraint. Because of the generous number of configurable delay elements in the datapath, it is straightforward to pipeline the computation to meet this path delay constraint. The multiplier is generally on the critical path, but the delay is well-balanced with that needed by other communication paths. In cases where feedback loops are present, the circuit will necessarily be C-slowed[9], that is, run at the $100 \mathrm{MHz}$ clock rate, but with sufficient additional registers so that results are produced every $\mathrm{N}$ cycles, where $\mathrm{N}>1$.

\subsection{Layout Area}

Table 3 presents the area of each the RaPiD-Benchmark components and the percentage of the cell area devoted to each part of the datapath. This area is given in units of mega- $\lambda^{2}$. The cell area of $56.35 \mathrm{M} \lambda^{2}$ converts to 5.07 $\mathrm{mm}^{2}$ for $\lambda=.3 \mu(0.5 \mu$ process $)$ and $2.25 \mathrm{~mm}^{2}$ for $\lambda=.2 \mu(0.35 \mu$ process $)$, assuming the same design rules.

The area figures are divided into three categories: computational units, configurable interconnect and control. Approximately one third of the area is allocated to each. Figure 10 shows the floorplan of a RaPiD-Benchmark cell. The top part of the array is devoted to multipliers and data memories, the middle part is the segmented interconnection structure overlaid on ALUs, registers and bus connectors. The bottom part is the control path and also contains the static configuration cells.

The straightforward interpretation of the results in Table 3 is that the configuration overhead approximately triples the area of the layout. But this analysis ignores many factors. On one hand, a hardwired circuit needs 
Table 3: Area of a RaPiD-Benchmark cell.

\begin{tabular}{||l|c|c|c|c||}
\hline Component & Area $\left(\mathrm{M} \lambda^{2}\right)$ & Number & Total Area $\left(\mathrm{M} \lambda^{2}\right)$ & $\%$ of cell Area \\
\hline data memories & 2.81 & 3 & 8.43 & $15.0 \%$ \\
multipliers & 5.16 & 1 & 5.16 & $9.2 \%$ \\
ALUs & 0.92 & 3 & 2.76 & $4.9 \%$ \\
general registers & 0.39 & 6 & 2.32 & $4.1 \%$ \\
\hline Functional Unit Subtotal & & & 18.67 & $33.1 \%$ \\
\hline \hline Multiplier/RAM I/O routing & & & 2.87 & $5.1 \%$ \\
Input multiplexers & 0.22 & 20 & 4.44 & $7.9 \%$ \\
Output drivers & 0.22 & 14 & 3.10 & $5.5 \%$ \\
Bus connectors & 0.39 & 15 & 5.90 & $10.5 \%$ \\
Configurable delays & 0.39 & 5 & 1.94 & $3.4 \%$ \\
\hline Configurable Interconnect Subtotal & & & 14.87 & $32.4 \%$ \\
\hline \hline Soft control bits & 0.07 & 104 & 6.89 & $12.2 \%$ \\
Programmable logic blocks & 0.35 & 3 & 1.05 & $1.9 \%$ \\
Bus connectors & 0.01 & 104 & 1.44 & $2.6 \%$ \\
SRAM configuration cells & 0.002 & 312 & 0.79 & $1.4 \%$ \\
Configuration memory overhead & & & 2.72 & $4.8 \%$ \\
\hline Control Subtotal & & & 12.89 & $22.9 \%$ \\
\hline Unused space & & & 6.54 & $11.6 \%$ \\
\hline Total cell area & & 56.35 & $100 \%$ \\
\hline
\end{tabular}

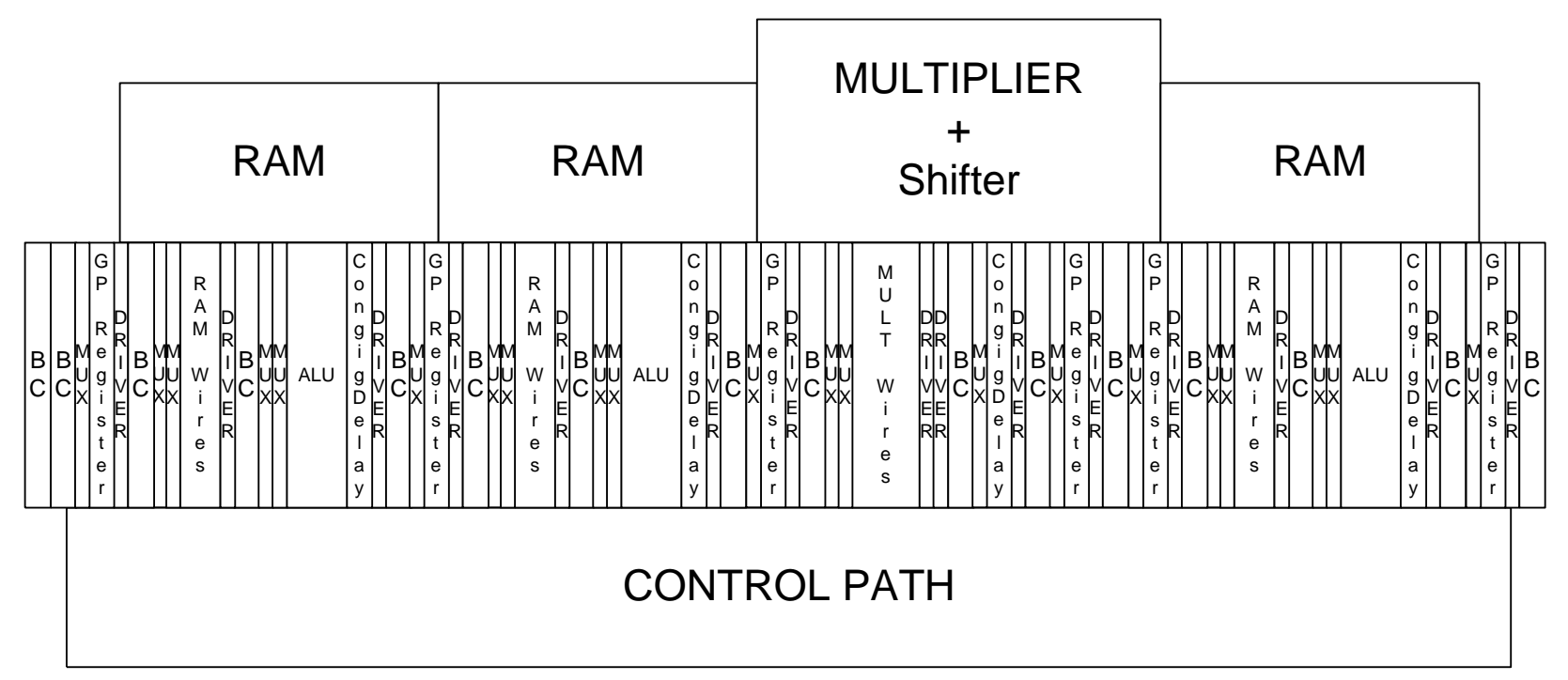

Figure 10: Floorplan of a RaPiD-Benchmark cell. 
both interconnect and some form of control, typically provided by FSMs, both of which are called overhead here. On the other hand, a hardwired circuit would typically not use all the functional units or the full data width. Even more perplexing is how to evaluate the ability of a configurable datapath to execute a variety of different computation while a fixed circuit executes only one. The approximately $67 \%$ overhead for RaPiD datapaths compares with the approximately 95-98\% overhead for FPGAs.

Table 4 estimates the area of the components needed outside the datapath itself. This area represents about $15 \%$ of the total area of a 16-cell array.

Table 4: Area of RaPiD Controller and Data Streams.

\begin{tabular}{||l|c|c|c|}
\hline Component & Area $\left(\mathrm{M} \lambda^{2}\right)$ & Number & Total Area \\
\hline Instruction generator & 30 & 1 & 30 \\
Address generators & 6 & 6 & 36 \\
16 entry data FIFOs & 1.5 & 6 & 9 \\
Inter-row bus connectors & 15 & 4 & 60 \\
\hline Total auxiliary area & & & 135 \\
\hline
\end{tabular}

\subsection{Power Consumption}

The $\mathrm{RaPiD}$ architecture has features that makes it attractive for low power applications. Communication in the linear array is done using relatively short buses and only units that are used by an application consume power. This is done by turning off the clock to registers that are configured out of the computation and tying inputs of unused functional units and buses to ground. Thus power is used only to drive the clock backbone and to clock the units actually performing computation and data communication.

Table 5a gives an estimate of the power consumption for various components in the datapath. These figures were derived using HSpice and PowerMill to analyze the layout. Each entry gives the average operating current used by the RaPiD component. Bus transfer refers to driving a value from the output of one functional unit, or bus connector, to the input of another via a long bus. (These figures do not give a true indication of possible low-power performance since our layout of the RaPiD components optimized for performance rather than power.)

Table 5: Power Consumption. (a) Average operating current of RaPiD components. (b) Application performance.

(a)

\begin{tabular}{||l|c|}
\hline Component & Power \\
\hline Multiplier & $23 \mathrm{ma}$ \\
ALU & $2 \mathrm{ma}$ \\
Memory read/write & $13 \mathrm{ma}$ \\
Register & $0.5 \mathrm{ma}$ \\
Bus transfer & $0.7 \mathrm{ma}$ \\
\hline
\end{tabular}

(b)

\begin{tabular}{||l|l|c|c|}
\hline Application & $\begin{array}{l}\text { Computational } \\
\text { bandwidth }\end{array}$ & $\begin{array}{c}\text { Memory } \\
\text { accesses/cycle }\end{array}$ & Power \\
\hline 16 tap FIR filter & 100M samples/sec & 2 & $1.9 \mathrm{~W}$ \\
1024 tap FIR filter & $1.56 \mathrm{M}$ samples/sec & 0.03 & $3.4 \mathrm{~W}$ \\
2D convolution, 4x4 kernel & $100 \mathrm{M}$ pixels/sec & 3 & $1.9 \mathrm{~W}$ \\
Matrix multiply & $763128 \times 128$ matrices/sec & 1.5 & $4.1 \mathrm{~W}$ \\
8x8 DCT/IDCT & $1.56 \mathrm{M}$ blocks/sec & 2 & $4.0 \mathrm{~W}$ \\
Full motion estimation & & & \\
$\quad 8 \times 8$ blocks, 24x24 window & 865K blocks/sec & $<0.1$ & $2.2 \mathrm{~W}$ \\
Peak power & & & $6.1 \mathrm{~W}$ \\
\hline
\end{tabular}

Table 5b shows the power consumed by the RaPiD datapath for a set of applications. If all components and buses were clocked, peak power would be $6.1 \mathrm{~W}$. These power figures illustrate the improvement that can be obtained by using configuration information to reduce power consumption. We emphasize that these numbers are only estimates.

\subsection{Application Performance Results}

This section presents the overall performance results for several applications that have been mapped to the RaPiD-Benchmark architecture. References [5] and [3] contain the details on how these applications are mapped to a RaPiD pipeline.

Matrix multiply can be performed on arbitrarily sized matrices. Once the pipeline is full (and assuming no memory stalls) RaPiD performs at a sustained rate of approximately 1.6 billion MACs (multiply-accumulates) per second. The precise performance depends on memory stalls, frequency of reconfiguration, matrix dimensions 
and tiling granularity. An average of 1.5 memory accesses (reads and writes) are performed per cycle. A relatively pedestrian memory system can keep up the pace for the mostly linear addressing performed by matrix multiply.

A 16-cell RaPiD array can efficiently compute an $8 \times 8$ 2D-DCT by performing two matrix multiplies in the datapath, passing the transposed output of the first multiply to the input of the second. For images larger than $256 \times 256$ pixels, RaPiD achieves a sustained rate of almost 1.6 billion MACs, including reconfiguration overhead between images, with an average of 2 memory accesses per cycle.

Motion estimation is a very compute-intensive application that is performed in conjunction with data compression of moving images. As with matrix multiply and DCT, RaPiD performs at a sustained rate of 1.6 billion difference/absolute value/accumulate operations per second but with an average of 0.1 memory accesses per cycle. This performance is achievable even for relatively small images.

Motion picture compression requires both motion estimation and DCT to be performed on each frame. Given a reconfiguration time of 2000 cycles ( $20 \mu \mathrm{sec}$.), little performance is lost to reconfiguration and pipeline filling for this application. For a standard $720 \times 576$ frame, the RaPiD-Benchmark architecture achieves about 12 frames per second when executing both full motion estimation and DCT (including 4000 reconfiguration cycles per frame and pipeline filling).

The performance and power results are summarized in Table $5 \mathrm{~b}$ for these and other applications.

\subsection{Comparison to Other Architectures}

RaPiD is most similar to systolic array architectures, which have been used for the past 20 years to solve computationally intensive problems. Most systolic arrays appear as ASICs, although some programmable systolic arrays have been defined, notably the Intel iWarp. Programmable systolic arrays use a very different control model based on the standard microprogrammed control of a general datapath. iWarp in particular closely resembled a microprocessor with hardware support for systolic communication. In contrast, RaPiD is much more fine-grained with small memories, configurable interconnect and a very efficient configurable control mechanism. RaPiD is clearly able to execute linear systolic algorithms, and in fact most RaPiD algorithms are systolic. But RaPiD can also be configured to implement algorithms that are not systolic, for example a Viterbi decoder and a spline generator pipeline.

$\mathrm{RaPiD}$ is somewhat similar to SIMD and vector architectures, which also use very short instructions relative to the number of operations being performed. RaPiD is similar to SIMD in that a single instruction is used to control all stages of the pipeline. But RaPiD is not restricted in the same way because of its configurable control path. For example, the RaPiD pipeline can be configured to perform two different computations in two different parts of the pipeline. RaPiD is also similar is some ways to vector architectures, where the data memories are viewed as distributed vector registers. However, these RaPiD memories do not have the high bandwidth to memory that vector registers do, and vector architectures do not take advantage of the local reuse of data and the fine-grained chaining inherent in systolic algorithms.

Quantitative comparisons to other architectures are difficult because of differences in technology, application details, data format, and memory systems. For comparison, we cite here performance results for a high-performance digital signal processor and one of the highest performance FPGA-based reconfigurable computing machines.

De Greef et al. derive a motion estimation algorithm highly optimized for DSP-style architectures [4]. In a case study of the $50 \mathrm{MHz}$ Texas Instruments TMS320C80 digital signal processor (containing four 32-bit DSPs and one 64-bit RISC processor), they show that 23 TMS320C80 chips can implement motion estimation of $720 \times 576$ pixel frames at 25 frames/second. (A $60 \mathrm{MHz}$ version would reduce this requirement to 12 chips).

The PAM $P_{1}$ is an FPGA-based reconfigurable computing machine consisting of 23 Xilinx XC3090 FPGAs, a $4 \mathrm{MB}$ local RAM, and a 100MB/s host bus. The PAM project has reported some of the best performance for configurable machines. A single PAM $P_{1}$ board can perform 2D-DCT at a rate of 1.4 GOPS (an OP is a multiply, add, subtract or shift)[2]. This section showed that RaPiD achieves 1.6 GOPS.

\section{Conclusion}

RaPiD represents an efficient configurable computing solution for regular computationally-intensive applications. By combining the appropriate amount of static and dynamic control, it achieves substantially reduced control overhead relative to FPGA-based and general-purpose processor architectures. Processors must devote resources to be able to perform irregular and unpredictable computations, while FPGAs must devote resources to construct unpredictable circuit structures. RaPiD is optimized for highly predictable and regular computations which reduces the control overhead. The assumption is that RaPiD datapaths will be integrated closely with a RISC 
engine on the same chip. The RISC would control the overall computational flow, performing the unstructured computations which it does best, while farming out the heavy-duty, brute-force computation to RaPiD.

One open question then is how to best incorporate RaPiD into a larger system comprising a general-purpose processor and a more general memory system. One approach is to treat it as a co-processor. We believe that RaPiD should be bound much more closely to a general-purpose processor. In this model, it would be viewed as a special functional unit of the processor with its own special path to memory that could include the processor cache where appropriate. In such a model, the granularity of the computation passed to RaPiD could be relatively small, and the configuration information could be contained in the instruction stream and decoded to configure the RaPiD datapath. Such a tight interaction would greatly increase the application domain of RaPiD. Processors incorporating a RaPiD array could be used for both general-purpose computing as well as compute-intensive applications like digital signal processing.

\section{Acknowledgments}

We would like to thank Larry McMurchie, Stefan Berg, Jason Secosky, and Jeff Weener for their contributions to the RaPiD project.

\section{References}

[1] J. M. Arnold, D. A. Buell, D. T. Hoang, D. V. Pryor, N. Shirazi, and M. R. Thistle. The Splash 2 processor and applications. In Proceedings IEEE International Conference on Computer Design: VLSI in Computers and Processors, pages 482-5. IEEE Comput. Soc. Press, 1993.

[2] P. Bertin, D. Roncin, and J. Vuillemin. Programmable active memories: a performance assessment. In Parallel Architectures and Their Efficient Use: First Heinz Nixdorf Symposium Proceedings, pages 119-30. Springer-Verlag, 1993.

[3] D. C. Cronquist, P. Franklin, S. G. Berg, , and C. Ebeling. Specifying and Compiling Applications for RaPiD. In Field-Programmable Custom Computing Machines (FCCM-98), 1998.

[4] E. De Greef, F. Catthoor, and H. De Man. Mapping real-time motion estimation type algorithms to memory efficient, programmable multiprocessor architectures. Microprocessing 83 Microprogramming, 41(5-6):409-23, 1995.

[5] C. Ebeling, D. C. Cronquist, P. Franklin, and S. Berg. Mapping applications to the rapid configurable architecture. In Field-Programmable Custom Computing Machines (FCCM-97), 1997.

[6] H. Kung. Let's design algorithms for VLSI systems. Technical Report CMU-CS-79-151, Carnegie-Mellon University, January 1979.

[7] M. Lam. Software Pipelining: An Effective Scheduling Technique for VLIW Machines. In SIGPLAN '88 Conference on Programming Language Design and Implementation, pages 318-328, 1988.

[8] P. Lee and Z. M. Kedem. Synthesizing linear array algorithms from nested FOR loop algorithms. IEEE Transactions on Computers, 37(12):1578-98, 1988.

[9] C. E. Leiserson and J. B. Saxe. Retiming synchronous circuitry. Algorithmica, 6:5-35, 1991.

[10] D. I. Moldovan and J. A. B. Fortes. Partitioning and mapping algorithms into fixed size systolic arrays. IEEE Transactions on Computers, C-35(1):1-12, 1986.

[11] J. E. Vuillemin, P. Bertin, D. Roncin, M. Shand, H. H. Touati, and P. Boucard. Programmable active memories: reconfigurable systems come of age. IEEE Transactions on Very Large Scale Integration (VLSI) Systems, 4(1):56-69, 1996. 